\title{
Lymph Node Ratio as a Good Prognostic Factor for Patients with Pathologic N3a Breast Cancer with 10 or More Metastatic Axillary Lymph Nodes
}

\author{
Seo Won Jung, M.D. ${ }^{*}$, Soo Young Lim, M.D. ${ }^{*}$, Yong Min Na, M.D. ${ }^{1}$, Young Jae Ryu, M.D. ${ }^{1}$, Jin Seong Cho, M.D. ${ }^{2}$, \\ Jung Han Yoon, M.D., Ph.D. ${ }^{1}$, Min Ho Park, M.D., Ph.D. ${ }^{1}$ \\ ${ }^{1}$ Department of Surgery, Chonnam National University Medical School and Chonnam National University Hwasun Hospital, Jeollanam-do; \\ ${ }^{2}$ Department of Surgery, Chonnam National University Medical School and Chonnam National University Hospital, Gwangju, Korea
}

Purpose: Pathologic N3a breast cancer is defined as having 10 or more metastatic axillary lymph nodes and metastases to the infraclavicular (level III axillary lymph) node. This study aimed to determine clinicopathological factors and assess the importance of lymph node ratio (LNR) as a prognostic factor for patient with N3a breast cancer treated with surgery without neoadjuvant chemotherapy. Methods: Medical records of 154 patients who underwent surgery were retrospectively analyzed. Patients were pathologically diagnosed with N3a breast cancer between May 2004 and December 2014. LNR was defined as the number of metastatic lymph nodes divided by the total number of resected lymph nodes. It was calculated using the receiver operating characteristic (ROC) curve. The median follow up period was 94 months (range, 10-205 months). Results: Among 154 patients with N3a breast cancer, 70 (45.5\%) had recurrence and 40 (26.0\%) died during the follow-up period. The 5 -year disease free survival (DFS) and overall survival (OS) rates after surgery were $63.0 \%$ and $85.9 \%$, respectively. LNR $>0.82$ (hazard ratio [HR]: 2.271; 95\% confidence interval [Cl]: 1.413-3.649; $p=0.001$ ) was a prognostic factor significantly associated with DFS. LNR>0.68 (HR: 2.156; 95\% Cl: 1.146-4.044; $p=0.017$ ) and invasive ductal carcinoma (HR: 0.125; 95\% Cl: 0.017-0.915: $p=0.041$ ) were significantly associated with OS. Conclusion: Although LNR values associated with DFS and OS are slightly different, LNR is a good prognostic factor for patients with N3a breast cancer.

Key Words: Advanced breast neoplasms, Lymph node ratio, N3a breast neoplasms

\section{INTRODUCTION}

Breast cancer is the second most common cancer and the most common newly diagnosed cancer among women in Korea [1]. Although the prognosis of breast cancer has been improving because of the development of adjuvant systemic therapies and early detection using screening mammography and ultrasonography, the proportion of patients with advanced breast cancer remains up to $8.6 \%$ in Korea [2]. Among these patients, a higher number of axillary lymph node metastases are associated with worse prognosis. According to the 8th

Correspondence: Min Ho Park, M.D., Ph.D.

Department of Surgery, Chonnam National University Medical School and Chonnam National University Hwasun Hospital, 322 Seoyang-ro, Hwasun-eup, Hwasun-gun, Jeollanam-do 58128, Korea

Tel: +82-61-379-7646, Fax: +82-61-379-7661, E-mail: mhpark@chonnam.ac.kr

*These authors contributed equally to this work.

This study was financially supported by Chonnam National University (Grant No. 2017-2833, 2019-0210).

Received: Aug 1, 2021 Revised: Aug 28, 2021 Accepted: Oct 7, 2021 edition of the American Joint Committee on Cancer (AJCC) tumor, node and metastasis (TNM) staging system, patients with 10 or more metastatic axillary lymph nodes who were classified as having pathologic N3a, regardless of T stage, were referred to having stage IIIC disease and had dismal prognosis compared with stage IV. Over several decades, the 5-year disease-free survival (DFS) rate has changed from $43 \%$ to $66 \%$ and the overall survival (OS) rate ranges from $58 \%$ to $81 \%$ for patients with pathologic N3a stage breast cancer [3-5].

The lymph node stage in the TNM staging system does not reflect the number of retrieved lymph nodes. Generally, surgeons consider removing more than 10 axillary lymph nodes during standard axillary lymph node dissection based on the quantitative classification of lymph node involvement. To evaluate the exact involvement of lymph nodes, the lymph node ratio (LNR) defined as the number of metastatic lymph nodes divided by the total number of retrieved lymph nodes has been reported as a prognostic factor for various malignancies $[6,7]$. Several studies have revealed that LNR can be helpful as a 
supplementary method for predicting prognosis in patients with breast cancer and higher LNR is associated with shorter DFS and OS [8-10]. Patients with pathologic N3a stage, albeit with a high LNR, could have better prognosis because of different pathologic or biomarker characteristics. Few studies have reported the prognostic role of LNR in patients with advanced breast cancer without neoadjuvant treatment. This study aimed to evaluate prognostic factors and determine the importance of LNR in patients with breast cancer with 10 or more metastatic axillary lymph nodes treated with surgery and subsequent systemic treatment.

\section{METHODS}

\section{Patients}

From May 2004 to December 2014, 247 patients with pathologic N3a stage breast cancer underwent surgery in Chonnam National University Hwasun Hospital. Exclusion criteria were as follows: 1) previous breast surgery, 2) neoadjuvant chemotherapy (NAC) or hormonal therapy, 3) distant metastases at first diagnosis, 4) bilateral or occult breast cancer, 5) male breast cancer patient, or 5) resection of internal mammary lymph node(s) or supraclavicular lymph node(s) simultaneously during the first breast surgery. After excluding 93 patients who met the exclusion criteria, 154 patients with pathologic N3a breast cancer were enrolled in this study. Our study was approved by the Institutional Review Board of our hospital, with the reference number CNUHH-2016-219. All patients were diagnosed with breast cancer through core needle biopsy or excisional biopsy and underwent breast magnetic resonance imaging and positron emission tomography (PET)-computed tomography CT before surgery. Patient data were collected through a retrospective review of medical records. Age; type of surgery; tumor size; T stage; histologic type and grade; resected and metastatic lymph nodes; estrogen receptor (ER), progesterone receptor (PR), and human epidermal growth factor receptor 2 (HER2) levels; lymphovascular invasion; chemotherapy; hormonal therapy; and radiotherapy were analyzed. Locoregional recurrence was considered when recurrent tumor was located in the parenchyma, chest wall, or skin of the ipsilateral breast or when metastatic lymph node(s) were detected in the ipsilateral axillary, supraclavicular, or internal mammary area. DFS was calculated as the period between a breast operation and the time when locoregional recurrence or dis- tant metastasis was detected. OS was calculated as the period between a breast operation and the time of death caused by breast cancer or the time of the last follow-up.

\section{Surgery}

When performing breast-conserving surgery (BCS), surgical margins were examined through frozen section analysis, including the upper, lower, outer, and inner parts of the excisional cavity. If the tumor was close to the nipple, the subareolar margin was examined. BCS was considered for all patients. However, mastectomy was performed for those who had multicentric disease, were expected to have cosmetic problems owing to a large mass, had invasion to the nipple, could not have negative surgical margins, or had undergone chest irradiation. The boundaries of mastectomy were the clavicle superiorly, rectus sheath inferiorly, sternal border medially, and latissimus dorsi muscle laterally.

If patients had suspicious metastatic axillary lymph node(s), fine needle aspiration cytology (FNAC) was performed for the axillary lymph nodes. Patients with pathologically confirmed lymph node metastasis underwent axillary lymph node dissection without sentinel lymph node biopsy. All lymph nodes of level I and level II were removed while preserving the long thoracic and the thoracodorsal nerves. Boundaries for axillary lymph node dissection were the axillary vein superiorly, pectoralis major superomedially, serratus anterior muscle inferiorly, and latissimus dorsi laterally. If lymph nodes were palpable in level II and level III, the pectoralis minor on the coracoid was divided to remove the lymph nodes in level III.

\section{Adjuvant therapy and follow up}

Almost all patients were treated with adjuvant chemotherapy using taxanes such as docetaxel $\left(75 \mathrm{mg} / \mathrm{m}^{2}\right)+\operatorname{adriamycin}\left(500 \mathrm{mg} / \mathrm{m}^{2}\right)+\mathrm{cy}$ clophosphamide $\left(500 \mathrm{mg} / \mathrm{m}^{2}\right)$ (TAC regimen) or adriamycin + cyclophosphamide followed by docetaxel (AC-T regimen). Only one patient refused adjuvant chemotherapy owing to performance status. Adjuvant radiotherapy was administered after TAC or between AC and T. In our institution, trastuzumab was available after 2010 based on HER2 results. Hormonal therapy was initiated after completing chemotherapy based on estrogen and progesterone receptors. An aromatase inhibitor or selective estrogen receptor modulator was selected according to menopause and the patient's levels of estradiol and folli- 
cle-stimulating hormone. Tests for tumor markers (carcinoembryonic antigen, carcinoma antigen 15-3) and imaging studies, such as breast ultrasonography, chest CT, and whole-body bone scan, were performed every 6 months after completing adjuvant chemotherapy and radiotherapy. In cases with meaningful increasing tumor-marker levels or abnormal findings on imaging studies, PET-CT was performed. If suspicious lesions were accessible, recurrence was confirmed through FNAC, biopsy, or surgical removal. If suspicious lesions were inaccessible, recurrence or metastasis was considered based on the results of imaging studies.

\section{Statistical analyses}

The receiver operating characteristic (ROC) curve analysis was used to determine the proper value of LNR. The Kaplan-Meier method and log-rank test were used to compare survival data with univariate analysis according to DFS and OS. For multivariate analysis, the Cox proportional hazards model was used for variables showing statistical significance in the univariate analysis. All statistical analyses were performed using SPSS version 25.0 (IBM Inc., Armonk, NY, USA). Statistical significance was considered when the $p$-value was less than 0.050 .

\section{RESULTS}

\section{Patients and characteristics}

All patients were female. Their median age was 50 years (range, 2777 years). Among 154 patients, 52 (33.8\%) underwent BCS and 102 (66.2\%) underwent mastectomy. The median tumor size was $3.1 \mathrm{~cm}$ (range, $0.3-11 \mathrm{~cm})$. The most common T stage was T2 (86 patients, $55.8 \%$ ), followed by T1 (37 patients, $24.0 \%$ ), T3 (25 patients, $16.2 \%$ ), and T4 (6 patients, 3.9\%). The median number of retrieved lymph nodes was 24 (range, 11-52). The median number of metastatic lymph nodes was 18 (range, 7-51). The number of patients with positive ER, PR, and HER2 was 90 (58.4\%), 81 (52.6\%), and 56 (36.4\%), respectively. There were 51 (33.1\%) patients with the triple-negative (TN) subtype. Most patients (136 patients, $88.3 \%$ ) had the histological subtype of invasive ductal carcinoma. Regarding histologic grade, 72 (46.8\%) patients had moderate grade, 75 (48.7\%) had high grade, and 7 (4.5\%) had low grade. Lymphovascular invasion was observed in 102 (66.2\%) patients. All patients, except one, were treated with adjuvant chemotherapy. A total of 149 (96.8\%) patients underwent radiotherapy. The median follow-up period was 94 months (range, 10-205 months) (Table 1).

\section{Recurrence and survival outcome}

Among 154 patients, 70 (45.5\%) had recurrence during follow-up, including 11 (15.7\%) who had only locoregional recurrence. Among the 56 patients who had distant metastases, 51 (72.9\%) had metastases

Table 1. Characteristics of 154 patients with pathological N3a breast cancer

\begin{tabular}{|c|c|}
\hline Characteristic & Value \\
\hline Age (yr) & $50(27-77)$ \\
\hline \multicolumn{2}{|l|}{ Breast operation } \\
\hline Breast conserving surgery & $52(33.8)$ \\
\hline Mastectomy & $102(66.2)$ \\
\hline Tumor size $(\mathrm{cm})$ & $3.1(0.3-11)$ \\
\hline \multicolumn{2}{|l|}{ pT classification* } \\
\hline $\mathrm{T} 1$ & $37(24.0)$ \\
\hline $\mathrm{T} 2$ & $86(55.8)$ \\
\hline $\mathrm{T} 3$ & $25(16.2)$ \\
\hline $\mathrm{T} 4$ & $6(3.9)$ \\
\hline Retrived lymph nodes & $24(11-52)$ \\
\hline Number of metastatic lymph nodes & $18(7-51)$ \\
\hline ER positive & $90(58.4)$ \\
\hline PR positive & $81(52.6)$ \\
\hline \multicolumn{2}{|l|}{ HER2 status } \\
\hline Negative & $98(63.6)$ \\
\hline Positive & $56(36.4)$ \\
\hline \multicolumn{2}{|l|}{ Molecular subtype } \\
\hline Triple negative & $51(33.1)$ \\
\hline Others & $103(66.9)$ \\
\hline \multicolumn{2}{|l|}{ Histology } \\
\hline Invasive ductal carcinoma & $136(88.3)$ \\
\hline Others & $18(11.7)$ \\
\hline \multicolumn{2}{|l|}{ Histologic grade } \\
\hline Low (Grade 1) & $7(4.5)$ \\
\hline Moderate (Grade 2) & $72(46.8)$ \\
\hline High (Grade 3) & $75(48.7)$ \\
\hline Lympho-vascular invasion & $102(66.2)$ \\
\hline \multicolumn{2}{|l|}{ Chemotherapy } \\
\hline Taxane based & $148(96.1)$ \\
\hline Non taxane based & $5(3.2)$ \\
\hline None & $1(0.6)$ \\
\hline Trastuzumab therapy & $31(20.1)$ \\
\hline Radiotherapy & $149(96.8)$ \\
\hline Hormonal therapy & $85(55.2)$ \\
\hline SERM & $55(35.7)$ \\
\hline Aromatase inhibitor & $30(19.5)$ \\
\hline Follow up (mo) & $94(10-205)$ \\
\hline
\end{tabular}

Values are presented as median (range) or number (\%).

$\mathrm{ER}=$ estrogen receptor; $\mathrm{PR}=$ progesterone receptor; $\mathrm{HER} 2=$ human epidermal growth factor receptor 2; SERM = selective estrogen receptor modulator. *The 8th edition of American Joint Committee on Cancer (AJCC) TNM classification. 
Table 2. Type of recurrence

\begin{tabular}{lc}
\hline Variable & No. $(\%)$ \\
\hline Loco-regional recurrence only & $11(15.7)$ \\
Single distant metastasis & $51(72.9)$ \\
Multiple distant mestastases & $5(7.1)$ \\
Loco-regional + distant metastases & $3(4.3)$ \\
Total & $70(100.0)$ \\
\hline
\end{tabular}

to one organ, $5(7.1 \%)$ had metastases in multiple organs, and $3(4.3 \%)$ had both distant metastases and locoregional recurrence (Table 2). The most common site of distant metastasis was the bone (22 patients, $14.1 \%)$, followed by the lungs and pleura (8 patients, 5.2\%). The 5 -year DFS and OS after surgery in all patients were $63.0 \%$ and $85.9 \%$, respectively (Figure 1).

\section{Variables related to DFS}

The calculated LNR according to recurrence using the ROC curve was 0.82 . The area under the curve was 0.669 . The sensitivity and specificity were $54.3 \%$ and $74.4 \%$, respectively. In the univariate analysis pathologic T stage (T1-2 vs. T3-4, $p=0.021)$ and LNR $(\leq 0.82$ vs. $>0.82, p<0.001)$ were significant factors associated with DFS. However, age, breast surgery type, estrogen receptor status, lymphovascular invasion, histologic type, histologic grade and molecular subtype (triple negative breast cancer [TNBC] vs. others) were not significant factors for DFS. Multivariate analysis showed that LNR $>0.82$ (hazard ratio [HR], 2.271; 95\% confidence interval [CI]: 1.413-3.649: $p=0.001)$ was a significant factor associated with recurrence, and pathologic T3-4 (HR: 1.665; 95\% CI: 0.979-2.832; $p=0.060$ ) was not a significant factor in the multivariate analysis (Table 3). Figure 2A shows disease-free survival curve according to the lymph node ratio (0.82 vs. $>0.82, p<0.001)$.

\section{Variables related to OS}

The LNR calculated according to survival using the ROC curve was 0.68 . The area under the curve was 0.643 . Its sensitivity and specificity were $75.6 \%$ and $52.2 \%$, respectively. In the univariate analysis, type of breast surgery (BCS vs. mastectomy, $p=0.042)$, LNR $(\leq 0.68$ vs. $>0.68, p=0.032$ ), and histologic type (invasive ductal carcinoma vs. others, $p=0.038$ ) were statistically significant factors for OS. However, age, pathologic T stage, estrogen receptor status, histologic grade, lymphovascular invasion, and molecular subtype (TNBC vs. others) were
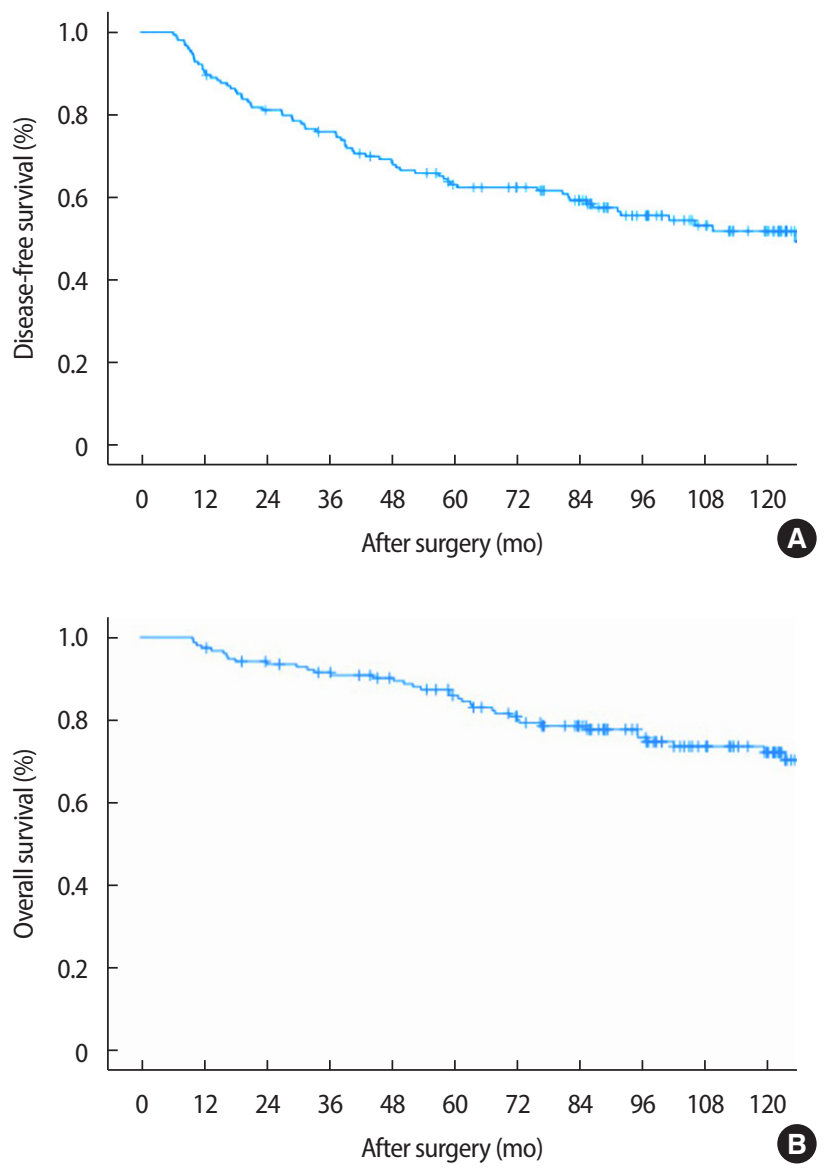

Figure 1. (A) Disease-free survival rates of 154 patients with pathological N3a breast cancer. (B) Overall survival rates of 154 patients with pathological N3a breast cancer.

not statistically significant factors for OS. Multivariate analysis showed that LNR > 0.68 (HR: 2.156; 95\% CI: 1.146-4.044; $p=0.017$ ) and histologic non-invasive ductal carcinoma type (HR: 0.125; 95\% CI: $0.017-0.915 ; p=0.041)$ were significant factors associated with OS, but mastectomy (HR: 2.065; 95\% CI: 0.947-4.504; $p=0.068$ ) was not significantly associated with OS (Table 4). Figure 2B shows overall survival curve according to the lymph node ratio ( 0.68 vs. $>0.68$, $p=0.032)$.

\section{DISCUSSION}

According to the 8th edition of the AJCC TNM classification, any T and N3 stage breast cancer without distant metastases is considered stage IIIC. The substantial burden of nodal disease in breast cancer is related to poor prognosis. Considering the presence of metastatic 
Table 3. Univariate and multivariate analyses associated with recurrence

\begin{tabular}{|c|c|c|c|c|}
\hline \multirow{2}{*}{ Variable } & \multicolumn{2}{|c|}{ Univariate } & \multicolumn{2}{|c|}{ Multivariate } \\
\hline & 5 years DFS (\%) & $p$-value & $\mathrm{HR}(95 \% \mathrm{Cl})$ & $p$-value \\
\hline Age (yr) & & 0.108 & & \\
\hline$<45$ & 51.8 & & & \\
\hline$\geq 45$ & 68.5 & & & \\
\hline Breast surgery & & 0.056 & & \\
\hline BCS & 72.5 & & & \\
\hline Mastectomy & 58.2 & & & \\
\hline pT classification* & & 0.021 & $1.665(0.979-2.832)$ & 0.060 \\
\hline $\mathrm{T} 1-2$ & 67.8 & & & \\
\hline T3-4 & 43.3 & & & \\
\hline LN ratio & & $<0.001$ & $2.271(1.413-3.649)$ & 0.001 \\
\hline$\leq 0.82$ & 69.3 & & & \\
\hline$>0.82$ & 53.3 & & & \\
\hline Histology & & 0.643 & & \\
\hline Invasive ductal carcinoma & 62.6 & & & \\
\hline Others & 65.8 & & & \\
\hline Histologic grade & & 0.475 & & \\
\hline $1-2$ & 65.1 & & & \\
\hline 3 & 60.9 & & & \\
\hline Lymphovascular invasion & & 0.486 & & \\
\hline Absent & 64.3 & & & \\
\hline Present & 62.4 & & & \\
\hline ER status & & 0.069 & & \\
\hline Negative & 54.4 & & & \\
\hline Positive & 69.2 & & & \\
\hline Molecular subtype & & 0.251 & & \\
\hline Triple negative & 56.4 & & & \\
\hline Others & 66.3 & & & \\
\hline
\end{tabular}

$\mathrm{DFS}=$ disease free survival; $\mathrm{HR}=$ hazard ratio; $\mathrm{Cl}=$ confidence interval; $\mathrm{BCS}=$ breast conserving surgery; $\mathrm{LN}=$ lymph node; $\mathrm{ER}=$ estrogen receptor. *The 8th edition of American Joint Committee on Cancer (AJCC) TNM classification.
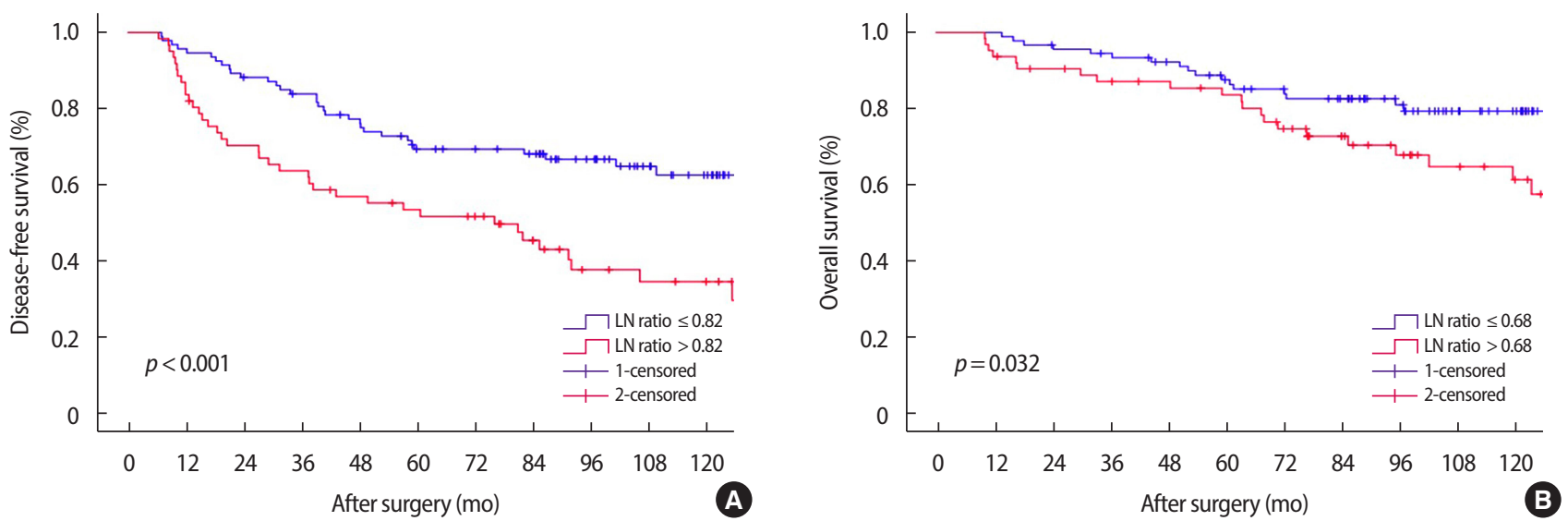

Figure 2. (A) Disease-free survival curve according to the lymph node ratio. (B) Overall survival curve according to the lymph node ratio.

lymph node lesions in stage IIIC breast cancer, not all patients with stage IIIC breast cancer have the same prognosis before surgery. Noh et al. [11] suggested that patients with clinical stage IIIC breast cancer involving both internal mammary lymph nodes and supraclavicular lymph nodes have worse survival outcomes than those who do not have the involvement of the two lymph nodes. Generally, clinicians 
Table 4. Univariate and multivariate analyses associated with survival

\begin{tabular}{|c|c|c|c|c|}
\hline \multirow{2}{*}{ Variable } & \multicolumn{2}{|c|}{ Univariate } & \multicolumn{2}{|c|}{ Multivariate } \\
\hline & 5 years OS (\%) & $p$-value & $\mathrm{HR}(95 \% \mathrm{Cl})$ & $p$-value \\
\hline Age (yr) & & 0.110 & & \\
\hline$<45$ & 81.9 & & & \\
\hline$\geq 45$ & 87.8 & & & \\
\hline Breast surgery & & 0.042 & $2.065(0.947-4.504)$ & 0.068 \\
\hline BCS & 88.1 & & & \\
\hline Mastectomy & 84.8 & & & \\
\hline pT classification* & & 0.070 & & \\
\hline $\mathrm{T} 1-2$ & 89.1 & & & \\
\hline T3-4 & 73.3 & & & \\
\hline LN ratio & & 0.032 & $2.156(1.146-4.044)$ & 0.017 \\
\hline$\leq 0.68$ & 87.5 & & & \\
\hline$>0.68$ & 83.6 & & & \\
\hline Histology & & 0.038 & $0.125(0.017-0.915)$ & 0.041 \\
\hline Invasive ductal carcinoma & 83.9 & & & \\
\hline Others & 100 & & & \\
\hline Histologic grade & & 0.335 & & \\
\hline $1-2$ & 92.1 & & & \\
\hline 3 & 79.1 & & & \\
\hline Lymphovascular invasion & & 0.757 & & \\
\hline Absent & 81.9 & & & \\
\hline Present & 87.9 & & & \\
\hline ER status & & 0.106 & & \\
\hline Negative & 77.5 & & & \\
\hline Positive & 91.9 & & & \\
\hline Molecular subtype & & 0.654 & & \\
\hline Triple negative & 80.0 & & & \\
\hline Others & 88.9 & & & \\
\hline
\end{tabular}

$\mathrm{OS}=$ overall survival; $\mathrm{HR}=$ hazard ratio; $\mathrm{Cl}=$ confidence interval; $\mathrm{BCS}=$ breast conserving surgery; $\mathrm{LN}=$ lymph node; $\mathrm{ER}=$ estrogen receptor. *The 8th edition of American Joint Committee on Cancer (AJCC) TNM classification.

tend to select NAC as the initial treatment for patients with suspicious metastatic supraclavicular or internal mammary lymph nodes, or even multiple level III axillary lymph nodes [12,13]. NAC might have the benefit of downstaging not only the size of the primary tumor but also the tumor burden of the lymph nodes. However, the current study did not include patients treated with NAC to exclude the effect of NAC. The recurrence and mortality of patients with N3a breast cancer have improved because of the development of taxane-based chemotherapy, endocrine therapy, and target therapy for decades. In the present study, the 5-year DFS and OS rates after surgery followed by adjuvant treatment were $63.0 \%$ and $85.9 \%$, respectively. These values are comparable to the results reported in previous studies on the survival rate of patients with N3a breast cancer [13-15].

It is well known that nodal involvement (the number and location of metastatic lymph nodes) is a significant prognostic factor for breast cancer after surgery [16]. Considering the number of metastatic axillary lymph nodes, N3 refers to having 10 or more metastatic axillary lymph nodes. Therefore, more than 10 axillary lymph nodes should be removed to assess the lymph node status. However, the TNM staging classification does not consider the number of retrieved lymph nodes. To reflect the number of retrieved lymph nodes, LNR, defined as the number of metastatic lymph nodes divided by the total number of retrieved lymph nodes, can be used to precisely predict prognosis in patients with equal $\mathrm{N}$ stage and might be a useful method for risk stratification. When patients have the same LNR, prognoses differ according to the value of the denominator or numerator. Furthermore, a high LNR might not necessarily mean perfect lymph node dissection, and a low LNR might not mean incomplete axillary lymph node dissection. Several studies have suggested various cut-off values (ranging from 0.2 to 0.65 ) for LNR in breast cancer, all of which showed that 
a high LNR was associated with low DFS and OS [10,16-18]. Some studies have revealed that LNR groups classified as low, intermediate, and high with each cut-off value have different survival outcomes, and patients with a high LNR have worse prognosis compared to those with a low LNR [10,19-21]. Sakin et al. [21] reported that LNR is a more useful prognostic factor than pathological lymph node staging for predicting survival in patients with node-positive breast cancer. In addition, Jin et al. [22] proposed using a modified LNR because it is superior to LNR and other lymph node staging systems especially in a limited LN harvest. The current study calculated different LNR values based on the ROC curve related to recurrence and survival. The LNR cut-off value for OS (0.68) was lower than that for DFS (0.82). Interestingly, patients with an LNR between 0.68 and 0.82 showed no significant difference in OS analysis. Different LNRs provide more information about treatment strategies compared with the previously mentioned studies.

ER, PR, and HER2 statuses are well-known prognostic and predictive biomarkers. With regard to DFS and OS, TNBC (negative ER, PR, and HER2) have more aggressive clinical outcomes and lack of choice therapeutic agents than other subtypes of breast cancer [23-26]. After the introduction of trastuzumab therapy (Herceptin ${ }^{\mathrm{TM}}$ ) for HER2 overexpressed subtype breast cancer, an effective treatment for TN breast cancer has not been established. In the current study, 51 (32.7\%) patients with the TN phenotype had no significant difference compared with non-TN breast cancer terms of DFS and OS. We believed the reason for this is that only 31 of 56 patients with HER2 overexpressed subtype were treated with trastuzumab therapy. Trastuzumab therapy based on HER2 status was approved by the Korea Ministry of Health and Welfare in 2010.

When making decisions about the aggressiveness of breast cancer, assessment of histologic grade including the composition of tubular and glandular formation, nuclear pleomorphism, and mitotic count is important. Several studies have shown that histologic grade is an independent and significant prognostic factor for breast cancer [27,28]. Schwartz et al. [28] reported that, as the tumor size and number of metastatic lymph nodes increased for each histologic grade, OS rates decreased. However, in our study, there was no significant difference in DFS and OS rates according to histologic grade. The surgical method may be used in conjunction with the T stage. Therefore, patients who were treated with BCS had better OS than those treated with mastectomy according to the univariate analysis.

This study had several limitations. First, the present study retrospectively enrolled a relatively small number of patients from a single institution. Second, some patients had inadequate biomarker information. Luminal A (positive ER or PR, negative HER2, Ki-67<14\%) and luminal B (positive ER or PR, negative HER2, Ki-67 $\geq 14 \%$; positive ER or PR, positive HER2) subtypes could not be classified owing to a lack of information about Ki-67 [29]. Third, there may have been a selection bias when choosing patients. Some patients with clinical N3a breast cancer were treated with NAC before surgery. Fourth, because trastuzumab was approved in 2010 by the Korea Ministry of Health and Welfare, this study included 56 patients with HER2 overexpression, including some, who received trastuzumab therapy and some who did not.

In conclusion, LNR can be used as a good prognostic factor for patients with $\mathrm{N} 3 \mathrm{a}$ breast cancer and 10 or more axillary metastatic lymph nodes. The LNR cut-off values associated with DFS and OS were slightly different. This study indicated that an $\mathrm{LNR}<0.82$ was associated with better DFS in patients with pathologic N3a breast cancer. With respect to OS, an $\mathrm{LNR}<0.68$ and histologic non-invasive ductal carcinoma type were good prognostic factors. Further research is needed to determine the prognostic factors for patients with pathologic stage N3a breast cancer patients treated with surgery followed by adjuvant systemic treatment.

\section{CONFLICT OF INTEREST}

The authors declare that they have no competing interests.

\section{REFERENCES}

1. Hong S, Won YJ, Lee JJ, Jung KW, Kong HJ, Im JS, et al. Cancer statistics in Korea: incidence, mortality, survival, and prevalence in 2018. Cancer Res Treat 2021;53:301.

2. Kang SY, Kim YS, Kim Z, Kim HY, Kim HJ, Park S, et al. Breast cancer statistics in Korea in 2017: data from a breast cancer registry. J Breast Cancer 2020;23:115.

3. Koca E, Kuzan TY, Dizdar O, Babacan T, Sahin I, Ararat E, et al. Outcomes of locally advanced breast cancer patients with $\geq 10$ positive axillary lymph nodes. Med Oncol 2013;30:615. 
4. Kim JM, Kim JY, Jung EJ, Kwag SJ, Park JH, Park T, et al. The prognosis factors among breast cancer patients with extensive axillary lymph node metastasis. Korean J Clin Oncol 2018;14:43-7.

5. Hur MH, Ko S. Metastatic axillary node ratio predicts recurrence and poor long-term prognosis in patients with advanced stage IIIC (pN3) breast cancer. Ann Surg Treat Res 2017;92:340.

6. Nusbaum DJ, Mandelbaum RS, Machida H, Matsuzaki S, Roman LD, Sood AK, et al. Significance of lymph node ratio on survival of women with borderline ovarian tumors. Arch Gynecol Obstet 2020;301:1289-98.

7. Liao Y, Yin G, Fan X. The positive lymph node ratio predicts survival in T1-4N1-3M0 non-small cell lung cancer: a nomogram using the SEER database. Front Oncol 2020;10:1356.

8. Cetin IA, Akay SU, Ozkok HBC, Sengoz M. Lymph node ratio as an independent prognostic factor for breast cancer-related mortality in patients with node-positive breast cancer. J Cancer Res Ther 2020; 16:1387.

9. He M, Zhang JX, Jiang YZ, Chen YL, Yang HY, Tang LC, et al. The lymph node ratio as an independent prognostic factor for node-positive triple-negative breast cancer. Oncotarget 2017;8:44870.

10. Tonellotto F, Bergmann A, de Souza Abrahão K, de Aguiar SS, Bello MA, Thuler LCS. Impact of number of positive lymph nodes and lymph node ratio on survival of women with node-positive breast cancer. Eur J Breast Health 2019;15:76.

11. Noh JM, Kim KH, Park W, Suh CO, Huh SJ, Choi DH, et al. Prognostic significance of nodal involvement region in clinical stage IIIC breast cancer patients who received primary systemic treatment, surgery, and radiotherapy. Breast 2015;24:637-41.

12. Spronk PER, van Bommel ACM, Siesling S, Wouters M, Peeters $\mathrm{MV}$, Smorenburg CH. Variation in use of neoadjuvant chemotherapy in patients with stage III breast cancer: results of the Dutch national breast cancer audit. Breast 2017;36:34-8.

13. Ai X, Liao X, Li J, Tang P, Jiang J. Clinical outcomes of N3 breast cancer: a real-world study of a single institution and the US surveillance, epidemiology, and end results (SEER) database. Cancer Manag Res 2020;12:5331.

14. Kim YY, Park HK, Lee KH, Kim KI, Chun YS. Prognostically distinctive subgroup in pathologic N3 breast cancer. J Breast Cancer 2016;19:163-8.

15. Grassadonia A, Vici P, Gamucci T, Moscetti L, Pizzuti L, Mentuccia
L, et al. Long-term outcome of breast cancer patients with pathologic N3a lymph node stage. Breast 2017;32:79-86.

16. Liu D, Chen Y, Deng M, Xie G, Wang J, Zhang L, et al. Lymph node ratio and breast cancer prognosis: a meta-analysis. Breast Cancer 2014;21:1-9.

17. Kim SW, Choi DH, Huh SJ, Park W, Nam SJ, Kim SW, et al. Lymph node ratio as a risk factor for locoregional recurrence in breast cancer patients with 10 or more axillary nodes. J Breast Cancer 2016; 19:169-75.

18. Kim J, Kim JH, Kim OB, Oh YK, Park SG. Clinical significance of the lymph node ratio in N1 breast cancer. Radiat Oncol J 2017;35: 227.

19. Hung M, Xu J, Nielson D, Bounsanga J, Gu Y, Hansen AR, et al. Evaluating the prediction of breast cancer survival using lymph node ratio. J Breast Cancer 2018;21:315-20.

20. De la Cruz-Ku GA, Chambergo-Michilot D, Valcarcel B, Rebaza P, Möller M, Araujo JM, et al. Lymph node ratio as best prognostic factor in triple-negative breast cancer patients with residual disease after neoadjuvant chemotherapy. Breast J 2020;26:1659-66.

21. Sakin A, Aldemir MN. Lymph node ratio predicts long-term survival in lymph node-positive breast cancer. Eur J Breast Health 2020;16:270.

22. Jin ML, Gong Y, Pei YC, Ji P, Hu X, Shao ZM. Modified lymph node ratio improves the prognostic predictive ability for breast cancer patients compared with other lymph node staging systems. Breast 2020;49:93-100.

23. Dent R, Trudeau M, Pritchard KI, Hanna WM, Kahn HK, Sawka CA, et al. Triple-negative breast cancer: clinical features and patterns of recurrence. Clin Cancer Res 2007;13:4429-34.

24. Fallahpour S, Navaneelan T, De P, Borgo A. Breast cancer survival by molecular subtype: a population-based analysis of cancer registry data. CMAJ open 2017;5:E734-9.

25. Gonçalves Jr H, Guerra MR, Duarte Cintra JR, Fayer VA, Brum IV, Bustamante Teixeira MT. Survival study of triple-negative and nontriple-negative breast cancer in a Brazilian cohort. Clin Med Insights Oncol 2018;12:1179554918790563.

26. Luo SP, Wu QS, Chen H, Wang XX, Chen QX, Zhang J, et al. Validation of the prognostic significance of the prognostic stage group according to the eighth edition of American Cancer Joint Committee on Cancer Staging System in triple-negative breast cancer: an analy- 
sis from surveillance, epidemiology, and end results 18 database. J Surg Res 2020;247:211-9.

27. Fidalgo F, Rodrigues TC, Pinilla M, Silva AG, Maciel Mdo S, Rosenberg $\mathrm{C}$, et al. Lymphovascular invasion and histologic grade are associated with specific genomic profiles in invasive carcinomas of the breast. Tumor Biol 2015;36:1835-48.

28. Schwartz AM, Henson DE, Chen D, Rajamarthandan S. Histologic grade remains a prognostic factor for breast cancer regardless of the number of positive lymph nodes and tumor size: a study of 161708 cases of breast cancer from the SEER program. Arch Pathol Lab Med 2014;138:1048-52.

29. Perou CM, Sorlie T, Eisen MB, van de Rijn M, Jeffrey SS, Rees CA, et al. Molecular portraits of human breast tumours. Nature 2000; 406:747-52. 